\title{
Un mundo de estructuras: simetrías grupo-teóricas desde el realismo infoestructural ${ }^{* \dagger}$
}

\author{
A World of Structures: Group-Theorethical Symmetries from Infostructural Realism
}

\author{
Javier Anta $a^{\ddagger}$
}

\begin{abstract}
Resumen
El realismo óntico-estructural, como una de las corrientes actuales de filosofía más relevantes a nivel internacional, defiende la idea de que el mundo lo constituyen estructuras físicas reales. French (2014) considera las simetrías físicas con la estructura primaria de la realidad. Ladyman y Ross (2007), por su parte, asumen que el mundo está constituido de por una estructura informacional no redundante. En este artículo defenderé la idea de que ambas posiciones no son incompatibles mediante una interpretación de las mencionadas simetrías fundamentales en tal estructuras ónticas que contienen y comprimen en ellas mismas toda la información física (entendida como complejidad Kolmogorov) del mundo.
\end{abstract}

Palabras clave: realismo estructural - simetrías - información

\begin{abstract}
Ontic Structural Realism, as one of the most current trends in philosophy of science and metaphysics, defends the idea that the world is ultimately constituted of real physical structures. French (2014) regards physical symmetries as the foundational structure of a world without objects. On the other hand, Ladyman and Ross (2007) hold that the world is essentially made of non-redundant informational structure. I will argue in this paper that these two positions are by no means incompatible, as I will show by interpreting French's physical symmetries as real structures encompassing/ compressing every piece of information (Kolmogorov complexity) within the world.
\end{abstract}

Keywords: structural realism - symmetries - information

* Recibido: 5 de febrero de 2018. Aceptado con revisiones: 17 de junio de 2018.

† El presente trabajo de investigación ha sido financiado mediante una beca FPU del Ministerio de Educación, Cultura y Deporte de España asociada al proyecto de investigación "Leyes, explicación y realismo en ciencias físicas y biomédicas" (FFI2016-76799-P). Así mismo, este trabajo ha sido realizado en el marco investigador el Grupo de Investigación en Filosofía Analítica LOGOS y la Universitat de Barcelona.

‡ Universidad de Barcelona - LOGOS, España. Para contactar al autor, por favor, escribir a: antajav@gmail.com/anta@ub.edu. Metatheoria 10(1)(2019): 1-11. ISSN 1853-2322. eISSN 1853-2330.

(C) Editorial de la Universidad Nacional de Tres de Febrero.

(C) Editorial de la Universidad Nacional de Quilmes.

Publicado en la República Argentina. 


\section{Introducción}

Quizás, sin mucho riesgo a caer en tópicos, el debate contemporáneo en torno al realismo científico sea una de las áreas temáticas de encuentro más comunes dentro de la filosofía general de la ciencia de las últimas décadas. Telegráficamente, el realismo científico consiste en la idea de que debemos, o al menos es recomendable, creer en aquellas entidades que presuponen nuestras "mejores" teorías científicas (e.g. aquellas con mayor poder explicativo, mayor éxito predictivo y/o más simples). Una ingente pluralidad de posiciones teóricas, desde el realismo más convencido de Psillos (1999) hasta el anti-realismo empírico de van Fraassen pasando por toda una gama intermedia de perspectivas, han florecido impetuosamente en los últimos años en respuesta a las varias problemáticas en torno a esta cuestión. El realismo estructural es una de las formas de realismo científico más importantes en el panorama filosófico actual.

En su variante radical o metafísica, el realismo "óntico-estructural” 1 afirma que nuestras mejores teorías científicas, en especial las teorías fundamentales físicas, nos muestran que el componente último de la realidad es estructural; por lo que los individuos/objetos no son más que manifestaciones secundarias de esta estructura o, en su forma radical, ni siquiera se le podría dotar de existencia a dichas entidades individuales. Todo lo que hay en el mundo es estructura. Dos de las posturas más radicales y a la vez consistentes dentro del realismo óntico-estructural las encontramos por un lado en el realismo grupoteórico de Steven French, en dónde las estructuras fundamentales de la realidad son simetrías físicas, y por otro lado en el realismo informacional de James Ladyman, el cuál sostiene que ciertos patrones de datos o estructuras informacionales constituyen los cimientos estructurales de la realidad.

Mi objetivo en este artículo es doble. Por un lado, reivindicar enérgicamente el valor del realismo óntico-estructural como filosofía de la ciencia en el contexto hispanohablante. Salvo el caso puntual del argentino Bruno Borge (2014), como único realista óntico-estructural de habla hispana que me consta, o Carlos Casado (2008) en tal que crítico del movimiento, no encontraremos ninguna línea de investigación grupal o individual en activo que se dedique a la difusión (por supuesto no existen traducción al castellano de las principales obras óntico-estructurales) y/o al desarrollo de una de las principales corrientes actuales a nivel mundial de filosofía de la ciencia y metafísica científica en dicha lengua. Lo cual es, en mi opinión, una derrota intelectual. Con este objetivo, dedicaré la segunda sección del presente artículo a delinear la evolución histórica del realismo estructural a finales del siglo XX y en las primeras décadas del siglo presente. ${ }^{2}$ Debido a su peso y complejidad, las perspectivas óntico-estructural de French y de Ladyman serán analizadas individual y comparativamente según sus particularidades a lo largo de la sección tercera y cuarta respectivamente.

Por otro lado, en la sección quinta pretendo establecer las bases de una perspectiva óntico-estructural que solvente los puntos débiles y sintetice las diferentes virtudes que encontramos en las posiciones radicales de Ladyman y French. En esta "tercera vía" óntico-estructural interpretaremos la ontología de French, basada en simetrías físicas fundamentales, desde la metafísica naturalizada informacionalestructuralista de Ladyman a partir de un análisis de tales simetrías como "mecanismos ónticos de compresión algorítmica”; o dicho de otro modo: tomando las simetrías físicas fundamentales realistamente, podemos asumir como estas contienen toda la información (en un sentido que aclararemos) del mundo. No hay realidad más allá de estos patrones de simetría primordial. Para aclarar esta tesis radical, deberemos comenzar por el principio.

\footnotetext{
${ }^{1}$ Borge traduce acertadamente "ontic structural realismo" en "realismo estructural óntico". Sin embargo, en este artículo optaré por "realismo óntico-estructural” para remarcar el estrecho lazo entre las estructuras y su realidad.

2 Para un análisis más en profundidad del recorrido histórico del realismo estructural tenemos en castellano la tesis doctoral de Borge (2014) o de primera mano el completo articulo para la SEP del propio Ladyman (2014).
} 


\section{Desarrollo finisecular del realismo óntico-estructural}

En su famoso artículo de 1989, John Worrall propuso el "realismo estructural" como respuesta al escepticismo generado por ciertos argumentos antirrealistas, especialmente el conocido como "metainducción pesimista": no debemos creer en las entidades que sugieren nuestras teorías científicas, en tanto que estas resultarán ser falsas en un futuro, al igual que todas las teorías que le han precedido. En concreto, este fuerte argumento antirrealista se dirige a un fenómeno central en la historia y filosofía historicista (Kuhn, Lakatos, Feyerabend, etc.) de la ciencia: la sustitución de una teoría por otra, con una ontología científica diferente susceptible de ser creída o no, dentro de una comunidad científica. La propuesta de Worrall es que, aunque la ontología de nuestras teorías científicas cambie debido a la sucesión de estas, hay algo que no desaparece en los cambios interteóricos: la estructura teórica. El caso paradigmático es el paso de la teoría del éter de Fresnel al electromagnetismo maxwelliano como explicaciones de la reflexión lumínica: mientras que en la primera teoría se propone el "éter" como entidad mecánica explicativa, en la segunda se rechaza por completo el "éter" como entidad/objeto real y se substituye por el "campo electromagnético". Se abre entonces el camino a una ontología de campos físicos, definida por los físicos estructuralistas Wigner y Weyl, y clave en el posterior realismo estructural de French o Ladyman, como veremos en las siguientes secciones. Pues bien, aunque la ontología científica cambie radicalmente en esta sucesión interteórica (la referencia del término "éter" se pierde), Worrall asume que la estructura constitutiva que representa la teoría de Fresnel queda preservada en la óptica maxwelliana, en tanto que existe una cierta similaridad sintáctica en las ecuaciones empleables en ambos casos; por tanto es esta estructura, y no las entidades, la que ha de ser tomada realistamente. La cuestión sobre qué tipo de estructura es la que se preserva quedó abierta. Esta corriente inicial de realismo "selectivo" (pues ha de seleccionar el fragmento de cada teoría en la que creer) en forma de realismo estructural reavivó en los 90s el debate del realismo científico, introduciendo en primera línea de discusión el complejo espacio entre realismo y antirealismo.

Otro de los puntos claves en la evolución intelectual del realismo estructural fue la distinción entre "realismo óntico-estructural" y "realismo epistémico-estructural", llevada a cabo por James Ladyman en su bien conocido artículo de 1998. El realismo óntico-estructural, en tal que postura metafísica, asume que aquella estructura referida por nuestras mejores teorías científicas es una estructura que existe de por sí en el mundo; de hecho, para el realismo óntico-estructural eliminativo (representado por French 1998), la estructura es precisamente lo único real, y no los individuos/objetos. Para el realismo epistémicoestructural, en tal que posición eminentemente epistémica, no son los individuos/objetos sino la estructura expuesta por nuestras mejores teorías lo único que podemos llegar conocer de la realidad. El realismo estructural de Worrall, e incluso las posiciones estructuralistas de relevantes figuras históricas como Cassirer, Weyl o Eddington, se enmarcarian dentro de esta última tipología. Además de remarcar esta distinción, Ladyman (1998) delinea diferentes tipologías de realismo óntico-estructural en función de los compromisos ontológicos que se puedan llegar a adoptar, desde la ya mencionada versión eliminativa (relaciones sin relata, fuertemente criticada por Psillos 1998) hasta una postura mucho más moderada en la que (i) la estructura o sistema de relaciones es lo ontológicamente fundamental y (ii) de la que las entidades individuales dependen en tal que nódulos de esa misma estructura (para un análisis más exhaustivo ver Ladyman; 2014). Uno de los artículos más significativos en esta línea fundacional del realismo óntico-estructural, aun antes de que el realismo estructural fuese popularizado por Worrall, es el análisis de la individualidad en el ámbito cuántico por Steven French (1989), supervisor doctoral del propio Ladyman, en el que pretende responder a la pregunta ¿Constituyen las partículas cuánticas entidades individuales? y en cuya respuesta justifica la primacía metafísica de la estructura sobre los objetos en la física moderna.

Durante principios del siglo XXI, el realismo óntico-estructural ganará un gran número de adeptos, todos ellos con un perfil más moderado (Esfeld 2004) que nuestros dos autores claves. Sin embargo, a 
pesar de lo variopinto de estas múltiples posiciones estructuralistas, todas ellas poseen un rasgo característico común, que se formulará ya explícitamente en Ladyman y Ross (2007), a saber: la estructura real primordial, y por lo tanto a la que más debemos comprometernos ontológicamente, es aquella que nos proporciona nuestras mejores (y más fundamentales) teorías físicas, como la Relatividad General o la Física de Campos Cuánticos. Por tanto, (a) fisicalismo o físico-centrismo, (b) fundamentalismo (en contraposición al anti-fundamentalismo de la Escuela de Stanford) y (c) realismo óntico-estructural formarán una indisociable unión en el desarrollo a principios de siglo de este último. Una vez llegado a este punto, cabe señalar las dos principales propuestas radicalmente óntico-estructurales, principales en términos de creatividad, contundencia teórica y coherencia sistemática, en el momento presente: (i) French con su realismo grupo-teórico, por un lado, y (ii) Ladyman, junto con Don Ross, en su realismo infoestructural, por otro lado. Por sus muchas particularidades y por el objetivo de este trabajo, nos detendremos en cada una de ella en las siguientes secciones.

\section{The Structure of the World: el realismo grupo-teórico de French}

La filosofía de la ciencia de Steven French constituye, con poco margen de duda, la versión contemporánea más madurada (y más prolongada, con casi cuatro décadas de desarrollo) de realismo óntico-estructural. En su opera magna tardía The Structure of the World (2014), la cual sintetiza gran parte de su labor filosófica, French deja claro las raíces históricas de su perspectiva: se nutre de las enseñanzas de los ya mencionados maestros estructuralistas de la primera mitad del siglo XX (Cassirer entre otros neokantianos, los físicos Weyl, Wigner o Eddington) y las reformula en nuestro contexto científico-filosófico actual en pos de los últimos avances empíricos y teóricos de las teorías físicas fundamentales.

Una de las ideas que articulan su filosofía de la ciencia es que la teoría de grupos (en diferentes ámbitos teóricos de la física), constituye el aparato formal que nos presenta o nos informa sobre cuál es la estructura real u objetiva del mundo. En el caso ilustrativo de la teoría de la relatividad especial, el llamado "grupo de Lorentz" o "grupo de simetrías lorentzianas" $\mathrm{O}(1,3)$ nos muestra cuales son los elementos espacio-temporales que cambian bajo ciertas transformaciones admisibles (por ejemplo un "boost" lorentziano o rotación espaciotemporal) y, en especial, aquellas estructuras que se mantienen invariables bajo estas mismas transformaciones. En el caso de las transformaciones de Lorentz, la estructura que se presenta invariable es la métrica minkowskiana del espacio-tiempo relativista (en la que se preserva, entre otras cosas, la velocidad de la luz con constante); esta sería, según el propio French, la estructura que nos presenta o nos informa de lo que debemos creer "realistamente" de la teoría de la relatividad general. Por esto, a la pregunta ¿Qué debemos creer de la teoría de, en este caso, la relatividad especial? No es ninguna entidad, como se asumiría en el realismo científico de individuos, sino propiamente la métrica de Minkwoski lo que se asumiría como la estructura que representa la realidad objetiva derivada grupoteóricamente de esta teoría en particular. Lo real en cada ámbito fundamental de la física es aquel sistema de relaciones que permanece invariable.

Sin embargo, para French hay algo ontológicamente más fundamental que, en el caso anterior, la métrica de Minkwoski en el ámbito especial-relativista, y es precisamente el grupo de simetrías que hacen posible la invariancia de esta estructura métrica bajo transformaciones de Lorentz. A parte de las simetrías espacio-temporales de Lorentz, las teorías físicas de mayor éxito empírico (como caso paradigmático tenemos el Modelo Standard de Partículas) se basan en un conjunto de "simetrías fundamentales", una por cada tipo de interacción fundamental, modeladas formalmente mediante los grupos lie $\mathrm{SO}(3)$, $\mathrm{SO}(2)$ y U(1). Como para otros autores (ver Lange 2007), las simetrías físicas fundamentales constituyen para French una suerte de "meta-leyes" de las naturaleza, que gobiernan y condicionan modalmente las leyes físicas de rango inferior. En este sentido, y como también será formulado en Ladyman y Ross (2007), el realismo grupo-teórico aboga por una ontología anti-fragmentalista (oponiéndose a Dupré o Cartwright) o 
unificacionista, por lo que para French una posiblemente futura "Teoría del Todo" o "Teoría de Campos Unificados” más allá del Modelo Standard de Partículas debería contener idealmente una super-simetría común a todos los ámbitos de la física fundamental, tanto a escenarios general-relativistas como cuánticos, que nos presentase teóricamente el elemento estructural último de la realidad. Esta idea podría ser formulada informalmente del siguiente modo:

(1) $\mathrm{SO}(3) \times \mathrm{SO}(2) \times \mathrm{U}(1) \times \mathrm{O}(1,3) \Rightarrow \mathrm{X}(\mathrm{N})$

siendo "X(N)" una representación irreducible del plausible grupo de super-simetrias. De hecho el propio French especula sobre cuál podría ser tal súper-simetría: su apuesta es la "Invarianza o Simetría Permutacional”, en la cual se basaría la distinción estadística fundamental entre partículas bosónicas (estadística Bose-Einstein) y fermiónicas (estadística Fermi-Dirac) por medio de la aplicación del Principio de Pauli en estados simétricos o anti-simétricos respectivamente, así como ciertas simetrías intrínsecas al espacio-tiempo relativista o a la Segunda Ley de la Termodinámica.

Aunque la bien conocida evidencia de ruptura espontanea de la simetría $\mathrm{SO}(2)$ parezca poner en jaque su posición, el autor resuelve esta tensión argumentando que no se produce una "ruptura" literal (ontológica) de tal simetría física, sino una degeneración en simetrías menores. Otra de las líneas rojas de esta concepción grupo-teórica es, tal y como French es consciente, la problemática de degenerar en posiciones platónico-pitagóricas al afirmar que la estructural fundamental de la realidad física es de naturaleza matemática (simetrías grupo-teóricamente formuladas). ¿Cómo establecer un criterio de demarcación que distinga una estructura matemática de una estructura propiamente física? Su estrategia no es otra que señalar el carácter semántico y negar la condición ontológica de dichas estructuras matemáticas: el grupo especial ortogonal $\mathrm{SO}(3)$ no es propiamente la simetría topológica en cuestión, sino el modo que tenemos de representar el contenido estrictamente físico del conjunto de simetrías fundamentales bajo la interacción fuerte. Ello lo plasma en el subtítulo Metaphysics and Representation de su obra, como una declaración explícita de intenciones. Aun asumiendo esta sutil distinción, como solución conclusiva deja mucho que desear, especialmente en cotas altas de abstracción teórica, tan frecuentes dentro de la física contemporánea. ¿Cómo distinguir cuando una subestructura perteneciente a sistema de estructuras compleja, como es el grupo lie $\mathrm{SO}$ (3) (que posee contenido algebraico abstracto y a su vez topológico-diferencial), contiene información física directa, o contiene información sobre otra estructura con contenido físico, o únicamente posee información sobre otra estructura matemática? La compleja situación parece rebasar el criterio semántico de demarcación físico-matemática de French. Dejemos ahora que hable el alumno.

\section{Every Thing Must Go: Ladyman y el realismo infoestructural}

La propuesta de realismo óntico-estructural de Ladyman, y en gran parte también la de Ross (aunque nosotros nos centraremos casi exclusivamente en Ladyman en lo que sigue), a pesar de confluir en ciertos puntos temáticos (ver Ladyman \& French 2002) tales como de la primacía ontológica y epistemológica de la física, se diferenciará notablemente de la visión global de su maestro. Primeramente, las estructuras fundamentales tras nuestras teorías científicas no serán dilucidadas grupo-teóricamente, como era el caso de French, sino información-teóricamente, o dicho de forma más simple, por medio de la teoría (postshannoniana) de la información, en un sentido que adelante especificaremos. Por ello mismo que a la corriente de realismo óntico-estructural que Ladyman defenderá, ya a partir de su exitoso Every Thing Must Go (2007), será denominada como "realismo infoestructural" (o "ITSR" por sus siglas en inglés). Dicha obra inaugura una nueva forma radicalmente diferente de hacer metafísica científica.

Otro ámbito clave en la separación intelectual de Ladyman frente a French es el énfasis de este primero en rechazar contundentemente lo que él denomina como "neo-escolástica" o metafísica analítica o 
apriorística en pos de una "metafísica naturalizada" o empírica en la que sean las ciencias naturales (sobre todo la física fundamental) las que legitimen el discurso metafísico y nos proporcionen el marco ontológico correspondiente; mientras que French, por su parte, enmarca su proyecto (no eminentemente naturalistico) de filosofía de la ciencia óntico-estructuralista dentro de esa misma metafísica analítica postQuine (en la que predominan discursos sobre los universales, disposicionalismo, estructuralismo modal, etc.) que tanto criticará su discípulo. Como mecanismo de garantía cientificista, Ladyman (2007) formula el "Principio de Cierre Naturalista", en la que toda tesis metafísica deberá ser empíricamente avalada por la conjunción de dos teorías físicas fundamentales. Se asumen incuestionablemente Relatividad General y Mecánica Cuántica (incluyendo Teoría de Campos Cuánticos) como dichas teorías fundamentales, aunque Ladyman y algunos físicos de prestigio como Asher Peres incluyen también Termodinámica, en tal prototeoría de sistemas, como el tercer pilar de la física. Dichas teorías muestran sin vacilación la primacía ontológica del "campo" o la "estructura de campo", tal y como se encuentra ya en Cassirer, frente al objeto o "individuo" de una manera u otra: en el caso cuántico, las partículas no son sino un valor significativo dentro de un cierto campo (bosónico o fermiónico, según el tipo de partícula), mientras que en el ámbito relativista, los puntos-individuos son consecuencia de relaciones topológicas en la variedad. En este sentido, el propio realismo estructural de Ladyman se deriva ideológica o meta-teóricamente de este hecho físico.

A pesar de su innegable naturalismo fisicalista, el programa filosófico de Ladyman pretende abarcar también un tratamiento de las ciencias particulares y los objetos-individuos en lo que él llama "Realismo Selvático", en oposición directa al Realismo Desértico (ontología minimalista) de Quine. Cómo pueda parecer, Quine se erige en el pensamiento de Ladyman como el máximo representante de una forma de hacer metafísica sin vigencia alguna en el presente. Mientras que para French la conexión entre física fundamental y ciencias especiales es una mera relación institucional que refleja especularmente la estratificación jerárquica de la realidad, Ladyman rechaza esta idea antediluviana de los estratos ontológicos o niveles jerárquicos de realidad, asumiendo que la estructura fundamental de la realidad deja "marcas" informacionales en cada ámbito de las ciencias particulares. Según él, distinguir estas marcas entre todo el paisaje científico es una tarea propia de la teoría de la información y de las ciencias de la complejidad.

El entrelazado e inconcluso esquema del Realismo Selvático puede ser esbozado como sigue: las ciencias especiales, en contra de la física fundamental, continuamente utilizan objetos/individuos en sus teorías. Estos son meros "objetos pragmáticos" sin valor ontológico alguno, pero que pueden llegar a referir o localizar (una vez "comprimidos información-teóricamente") lo que Daniel Dennett denominó "patrones reales". Es importante remarcar que estos patrones reales nos informan, desde cualquier ámbito en el que se encuentren, acerca de la estructura fundamental de la realidad. Desde dentro de las ciencias especiales los patrones reales son proyectados en objetos-individuos (cuya naturaleza es únicamente instrumental), por lo que la estructura fundamental del mundo solo se presenta de manera muy indirecta y mediada en dichas disciplinas; mientras que en la física fundamental, por otro lado, los patrones reales son directa y desnudamente representados en los ya mencionados campos. Ladyman abandona el vocabulario reduccionista, aún presente en Frech, para explicar la relación entre disciplina fundamental y disciplinas especiales en pos de una terminología "proyectabilista" del contenido teórico estas últimas sobre el contenido de la primera.

Si para French las estructuras fundamentales de la realidad quedan representadas en las simetrías que nos muestran nuestras mejores teorías físicas por medio de la teoría de grupos, para Ladyman, por otra parte, las estructuras fundamentales son estructuras información-teoréticas (es significativo saber que Ladyman asume la información, en un sentido preciso, como un fenómeno intrínsecamente físico) pero no especifica mucho más el tipo de estructura informacional, al menos hasta su capítulo "The World in the Data" dentro del volumen Scientific Metaphysics (2013). En esta publicación se solventará la 
mencionada falta de claridad con el deslumbrante titular para-wittgensteiniano: "El mundo es la totalidad de datos estadísticos no redundantes" (p. 111). Esto deja una cuestión abierta: ¿constituye la totalidad de datos estadísticos no redundantes la estructura informacional fundamental de la realidad buscada en Every Thing Must Go?

Aunque muchas (y significantes) son las diferencias ontológicas e ideológicas entre discípulo y maestro óntico-estructuralista, entre Ladyman y French, muchos son también los puentes que se pueden trazar entre el pensamiento de ambos. En la siguiente sección buscaremos esta plausible (y deseable) síntesis sobre la que trazar una vía intermedia de realismo estructuralista.

\section{El papel infoestructural de las simetrías grupo-teóricas}

Como punto de partida de esta sección sería conveniente que el lector se preguntase ¿Existe algún punto de convergencia entre estas dos cosmovisiones estructuralistas radicales ya vistas? Y sobre todo ¿Sería conveniente encontrar dicha síntesis? En forma de repuesta afirmativa a ambas preguntas, a continuación me dispondré a mostrar cómo sería tanto posible como deseable esta convergencia. Partiendo de una evaluación inicial, podríamos señalar que Ladyman combina una interesantísima metafísica naturalista con una ontología informacional indefinida, mientras French, por su lado, define escrupulosamente su ontología invariantista en un marco metafísico analítico tradicional. Defenderé que la posición ónticoestructural óptima, provendría de combinar una sofisticada metafísica naturalista que integre metodología no apriorística con una definida (o al menos definible) ontología invariantista-informacional. Mi propuesta no es otra que interpretar la ontología grupo-teórica frenchiana desde la metafísica informacional ladymaniana, no como un mero ejercicio interpretativo sin valor filosófico alguno, sino como una muestra de que el realismo óntico-estructural radical y maduro no se agota exclusivamente en estas dos opciones (aunque de hecho pueda nutrirse de ambas) y de que aún tiene muchísimo que aportar, no solo al debate del realismo científico, sino a la filosofía de la ciencia en general en nuestro contexto presente. Esa es la motivación que sustenta esta propuesta.

\subsection{La complejidad algorítmica de las teorías científicas}

Tomemos como caso la simetría gauge que emerge del grupo lie especial unitario tridimensional "SU(3)" tras la fuerza o interacción fuerte, estudiada en la teoría de campos gauge (o también de "recalibración" en castellano), que es aquel tipo de interacción física fundamental existente entre quarks que hace posible la estabilidad interna de partículas hadrónicas como protones y neutrones, impidiendo la desintegración repentina de los núcleos atómicos. Lo epistemológicamente dificultoso de este tipo de simetría gauge es que no es posible de ser imaginada, representada o concebida como algo más allá de una abstracción formal, pues la manera más intuitiva de representarla sería en una esfera riemanniana pentadimensional incrustada en tres dimensiones complejas (es decir, lo que sería equivalente a ni más ni menos que doce dimensiones habituales). De ahí que mantener una actitud realista acerca de lo que SU(3) representa constituya un acto de extravagancia epistémica. Tanto la Cromodinámica Cuántica como la Teoría de Campos Gauge muestran empíricamente como dicha estructura simétrica contenida en SU(3), a pesar de su enorme grado de contraintuitividad, ha de constituir ontológicamente uno los fundamentos físicos de la realidad; de otra forma, la carga cromática (la carga propia a la interacción fuerte) perteneciente a quarks y transmitida por gluones no se conservaría, y en consecuencia todos núcleos atómicos estables serían (meta)físicamente imposibles dentro nuestro mundo.

French nos diría que SU(3) nos muestra o contiene una simetría fundamental de la realidad, a la que como realistas estructurales debemos estar ontológicamente comprometidos; Ladyman, que la complejidad algorítmica de SU(3) muestra un patrón real. ¿Están refiriéndose a cosas (bueno, cosas no, a "estructuras") 
distintas? La complejidad algorítmica o complejidad de Kolmogorov, que desempeña un papel central en Ladyman, no es otra cosa que una forma de medir la cantidad de información que contiene un fragmento de realidad; en sentido estricto, es el mínimo número de símbolos necesarios para describir ese fragmento. Se ilustra paradigmáticamente con

(2) 01010101010101010101

(3) 1011101000101100

en tanto que, a pesar de que (2) contiene más cantidad de símbolos binarios que (3). En (2) encontramos lo que podríamos definir en términos de un "patrón" recurrente, de modo que "01" se repite once veces en toda la serie, una vez cada dos dígitos; mientras que en (3) la serie binaria es aparentemente aleatoria, por lo que no contiene ningún patrón aparente. Es decir, mientras que (3) es más algorítmicamente compleja o contiene más información, entendiendo aquí "información" a la von Neumann, en tal que la cantidad de operaciones o pasos algebraicos necesarios para describir el orden existente en un conjunto de datos (simplistamente: a menos orden más información); en la secuencia (2) se nos muestra la existencia una regularidad u orden a lo largo de una distribución distinta de los mismos componentes binarios. La idea subyacente en el planteamiento ladymaniano es que las teorías de la física fundamental actúan como programas algorítmicos que representan y computan fragmentos de realidad similares a la secuencia (2), mientras que las teorías de las ciencias especiales son más proclives a procesar secuencias morfológicamente afines a (3) como por ejemplo

(4) $0120130102101 \ldots$

Sin embargo, aunque bajo un algoritmo o programa teórico "B" no perteneciente a teorías fundamentales (nótese la codificación no binaria en (4)), digamos por ejemplo "bioquímico", se pudiese obtener un patrón dentro la serie (4), Ladyman asume que toda secuencia y todo patrón-regularidad obtenida en ella puede ser tomada como proyección de una secuencia ontológicamente más fundamental, derivable por un algoritmo teórico "F" de la física de Campos Cuánticos o General-Relativista. De este modo:

(4b) (4): $0120130102101401 \ldots$ es proyectable en $\Rightarrow(2): 0101 \ldots$

Una vez (4) ha sido algorítmicamente comprimido en un fragmento de (2), tal y como muestra en (4b), un programa teórico "F" podría dilucidar el "patrón real" que contiene dicha secuencia, mientras otro programa especial "B" no. Ello implica además, en un nivel meta-teórico, el paso de un código (derivado) digital a un código (fundamental) binario. La clave en esta maniobra meta-teórica de Ladyman es que la fundamentalidad científica contenida en secuencias similares a (2) reside en su "incompresibilidad". El grupo lie SU(3), que hemos utilizado anteriormente como ejemplo, sería por tanto el equivalente al módulo "01" en tal que patrón real perteneciente a la secuencia (2), derivada a su vez por un algoritmo teórico "F" de la Cromodinámica Cuántica. Esto constituiría la complejidad algorítmica de SU(3).

\subsection{Compresión informacional en las simetrías físicas. Reducción y proyección}

Lo interesante aquí es que en (2), lo patrones existentes son patrones "simétricos", en la que existe una suerte de simetría permutacional entre cada par de dígitos. Justo en este sentido y aunque no lo exponga en estos términos info-naturalísticos, también para French la física fundamental sería la única disciplina científica capaz de general series más morfológicamente próximas a (2) que a (3) o a (4), no por la incompresibilidad algorítmica de (2) sino por el hecho de (2) contiene simetrías irreducibles: nótese que las simetrías de (4) si son reducibles a las simetrías de (2). Recordemos que para el propio French la "simetría permutacional”, aquellas contenida en las series $(2,4)$ es aquel tipo de simetría que subyacería plausiblemente a otras simetrías más específicas. En el programa óntico-estructuralista frenchiano sí que cabe hablar de una reducción efectiva del contenido teórico de (4) en el contenido teórico de (2), mientras 
en el realismo infoestructural o "Selvático" sería más conveniente afirmar que la información semántica contenida en (2) se proyecta sobre la información semántica de (4), lo que posibilita la compresión algorítmica de (4) en (2).

Ha de quedar claro, antes de proseguir con nuestro análisis que la compresión algorítmica inter-teórica (Ladyman) no es el mero equivalente naturalístico de la reducción inter-teórica tradicional (French): en el primer caso las ciencias especiales tienen legitimidad algorítmica para localizar estructuras informacionales que remitan proyectivamente a patrones reales propios de la física fundamental, mientras que para French, a pesar de buscar extender su óntico-estructuralismo a las ciencias especiales (sin gran éxito, en mi opinión), su reduccionismo fisicalista va acompañado de un fuerte barniz eliminativista que impide cualquier tipo de legitimidad epistemológica a las ciencias especiales.

Desde una posición óntico-estructural, podemos decir que una simetría real constituye un mecanismo óntico (si se quiere podemos interpretarlas desde la noción de "mecanismo nomológico" de Nancy Cartwright) que consiste, naturalísticamente, en comprimir información redundante del mundo físico. Es importante diferenciar entre simetrías ónticas y epistémicas, simetrías propias de la realidad y simetrías propias de nuestros instrumentos teóricos. Un caso de simetría epistemológica serían las identidades de Bianchi, las cuales juegan un papel decisivo en la teoría de la Relatividad General; sin embargo dichas simetrías son propiedades que poseen los tensores riemannianos de curvatura como instrumentos teóricos, por lo que no algo que acontezca en la realidad física. Quizás para un realista epistémico-estructural las identidades de Bianchi constituirían un conjunto de simetrías fundamentales en el contexto generalrelativista, pero nunca para un realista óntico-estructural; para este último perfil de realista científico encontraríamos otro tipo de simetrías que no pertenecen idiosincráticamente a nuestras herramientas teoréticas sino a la realidad física, como son las ya mencionadas simetrías lorentzianas $\mathrm{O}(1,3)$ pero únicamente a nivel local. Como tesis clave de este trabajo defenderé que el conjunto de dichas simetrías ónticas fundamentales (es la función de la física el determinar empíricamente cuáles son dichas simetrías) contienen o comprimen toda la información del universo.

El ejemplo más simple para entender el rol infoestructural de las simetrías ónticas es la simetría especular o reflectiva. ${ }^{3}$ Imaginemos un escenario físico en que tenemos una partícula con ciertas propiedades definidas y otra partícula especularmente-simétrica con respecto a la primera; pues bien, podemos decir desde una perspectiva ladymaniana que la información algorítmica total relativa al escenario físico completo (dos partículas) está contenida o comprimida en una única partícula más la simetría especular. Supongamos ahora que tenemos un escenario físico con cualquier número finito de entidades con las mismas propiedades, digamos 7.923.684.244 partículas, y un conjunto de simetrías ónticas, obtenidas por translación y rotación sobre dimensiones espaciales (conocido como grupo de simetrías euclideas), sobre dichas entidades de modo arbitrario; pues bien, toda la información algorítmica o toda la complejidad Kolmogorov de este hipotético universo esta, de hecho, comprimida en una única partícula y en el grupo de simetrías. Equivalentemente, toda la información de (2) se encuentra ya contenida en "01" más en la simetría permutacional sobre cada dos dígitos consecutivos.

En esta línea argumental, uno de los grandes físicos del S.XX, John Wheeler, discípulo intelectual de Weyl (que a su vez es uno de los principales físico-matemáticos que inspiraron el realismo estructural), desarrolló la idea de que el universo solo necesitaba de la existencia de un único electrón, junto con un grupo apropiado de simetrías, para que fuese descriptible y explicable en términos de electrodinámica cuántica, que es la teoría fundamental que explica la interacción foto-electro-magnética. Lo curioso que para los estructuralistas radicales no es necesario postular si quiera un único electrón (signifique lo que signifique "único" aquí) en todo el universo, sino un campo electromagnético cuántico cuya estructura es la simetría óntica U(1). Una ontología de campos hace posible fundamentar la realidad física en la

\footnotetext{
${ }^{3}$ Nótese que el prefijo "S" en $\mathrm{SO}(3)$ y $\mathrm{SO}(2)$ significa precisamente que ese conjunto de simetrías carece de simetría reflectiva o especular.
} 
estructura (gauge) simétrica de ciertos campos sin necesidad de postular otras entidades. ${ }^{4}$ Diríamos que la complejidad Kolmogorov o información electromagnética de todo el universo está algorítmicamente comprimida en la ontología de la simetría lie U(1).

En términos del realismo infoestructural, cada campo electromagnético, cada único electrón (como partícula fermiónica) o fotón (como partícula bosónica) posee información electromagnética redundante. Desde una óptica realista, estas entidades/objeto no constituirían más que proyecciones informacionales de U(1) en tal que un único patrón real que se despliega a lo largo del universo en cada fenómeno afectado por la interacción electromagnética. No hay contenido informacional propiamente electromagnético más allá de U(1) y su inter-relación con las otras simetrías físicas fundamentales. Todo el mundo, en su pleno sentido ontológico, queda física-informacionalmente comprimido o contenido en las simetrías físicas fundamentales.

\section{Conclusión}

Como punto final a nuestra labor, remarcaríamos que el grupo de simetrías físicas fundamentales (asumiendo, como hicimos en la sección tercera, que la ciencia actual no nos proporciona una única simetría "X(N)" sino un conjunto de ellas " $\mathrm{SO}(3) \times \mathrm{SO}(2) \times \mathrm{U}(1) \times \mathrm{O}(1,3)$ "), constituye el único conjunto de estructuras algorítmicamente incompresibles de la realidad. En dichos "patrones estructurales" que son las simetrías físicas fundamentales confluye de manera bastante satisfactoria, según el análisis esbozado hasta aquí, la ontología simetricial de French en la metafísica naturalista de Ladyman, evitando tanto el exceso de metodología metafísica analítica del primero y la excesiva indefinición del segundo. Cualquier otro fenómeno real contiene información redundante relativa a dichas estructuras, basándose en esta idea Ladyman afirma que las ciencias especiales manipulan, más o menos indirectamente, información proveniente proyectualmente de estos patrones estructurales.

Si bien nuestro modesto objetivo de traducir la ontología de French al vocabulario ladymaniano como síntesis de los dos pilares óntico-estructuralistas ha quedado patente, el mayor y principal motivo que nos ha traído hasta aquí se ha llevado implícitamente a cabo en la consecución del primero: mostrar, aunque sea brevemente, algunas de las virtudes de una de las corrientes filosóficas más decisivas en el panorama intelectual actual, como lo es el realismo óntico-estructural más radical, en un contexto en el que inmerecidamente no tiene peso alguno: la academia hispanohablante.

Aunque queda mucho camino que trazar y mucha estructura que descubrir.

Bibliografía

Borge, B. (2014), "Los orígenes del realismo estructural: rastreando la tradición estructuralista en filosofía de la ciencia”, Principia 18(3): 295-322.

Casado, C. (2008), "El realismo estructural a debate: matemáticas, ontología y representación”, Revista de Filosofía 33(2): 49-66.

Esfeld, M. (2004), "Quantum Entanglement and a Metaphysics of Relations", Studies in History and Philosophy of Science Part B: Studies in History and Philosophy of Modern Physics 35(4): 601-617.

\footnotetext{
${ }^{4}$ Nótese que nuestra postura no deriva en una suerte de pitagorismo precisamente porque nuestro realismo se dirige a las estructuras informacionales físicas y no hacia las estructuras matemáticas: no creemos realistamente en U(1) en tal que un "haz fibrado", como estructura matemática que representa la simetría física del electromagnetismo, sino propiamente en la simetría física fundamental del electromagnetismo como el contenido informacional de U(1).
} 
Esfeld, M. y V. Lam (2006), “Moderate Structural Realism about Space-Time”, Synthese 160(1): 27-46.

French, S. (2014), The Structure of the World: Metaphysics and Representation, Oxford: Oxford University Press.

French, S y M. Redhead (1988), "Quantum Physics and the Identity of Indiscernibles", British Journal for the Philosophy of Science 39(2): 233-246.

French, S. y J. Ladyman (2003), "Remodelling Structural Realism: Quantum Physics and the Metaphysics of Structure", Synthese 136(1): 31-56.

Ladyman, J. (1998), "What is Structural Realism?", Studies in History and Philosophy of Science Part A 29(3): 409-424.

Ladyman, J. (2014), "Structural Realism”, en Zalta, E. N. (ed.), The Stanford Encyclopedia of Philosophy (Spring 2014 Edition), https://plato.stanford.edu/archives/spr2014/entries/structural-realism/.

Ladyman, J. y D. Ross (2007), Every Thing Must Go: Metaphysics Naturalized, Oxford: Oxford University Press.

Ladyman, J. y D. Ross (2013), "The World in the Data”, en Ross, D., Ladyman, J. y H. Kincaid (eds.), Scientific Metaphysics, New York: Oxford University Press, pp. 108-150.

Roberts, W. (2011), "Group Structural Realism”, British Journal for the Philosophy of Science 62(1): 47-69.

Psillos, S. (1999), Scientific Realism: How Science Tracks Truth, London/New York: Routledge. 